\title{
A Longitudinal Study of Industrial and Clerical Workers: Incidence of Carpal Tunnel Syndrome and Assessment of Risk Factors
}

\author{
Nancy Gell, ${ }^{1,2}$ Robert A. Werner, ${ }^{1,2,3,4,6}$ Alfred Franzblau, ${ }^{3,4,5}$ \\ Sheryl S. Ulin, ${ }^{4}$ and Thomas J. Armstrong ${ }^{3,4}$
}

This study followed workers over an extended period of time to identify factors which may influence the onset of Carpal Tunnel Syndrome (CTS). The purpose was to evaluate incidence of CTS and to create a predictive model of factors that play a role in the development of CTS. This prospective study followed 432 industrial and clerical workers over 5.4 years. Incident cases were defined as workers who had no prior history of CTS at baseline testing and were diagnosed with CTS during the follow-up period or at the follow-up screening. On the basis of logistic regression, significant predictors for CTS include baseline median-ulnar peak latency difference, a history of wrist/hand/finger tendonitis, a history of numbness, tingling, burning, and/or pain in the hand, and work above the action level of the peak force and hand activity level threshold limit value. This longitudinal study supports findings from previous cross-sectional studies identifying both work related ergonomic stressors and physical factors as independent risk factors for CTS.

KEY WORDS: carpal tunnel syndrome; occupational diseases; median nerve; cumulative trauma disorders.

\section{INTRODUCTION}

Upper extremity (UE) cumulative trauma disorders, specifically carpal tunnel syndrome (CTS), continue to account for the majority of reported occupational disorders. Current prevalence rates for CTS are estimated to be $1-5 \%$ in the general population $(1,2)$, depending on how CTS is defined, and $5-15 \%$ in the industrial setting (3-5). Retrospective studies have estimated the incidence of CTS to be 1.74 per 1000 person-years in the

\footnotetext{
${ }^{1}$ Department of Physical Medicine and Rehabilitation, University of Michigan Health System, Ann Arbor, Michigan.

${ }^{2}$ Department of Environmental Health Sciences, School of Public Health, University of Michigan, Ann Arbor, Michigan.

${ }^{3}$ Veteran Affairs Medical Center, Ann Arbor, Michigan.

${ }^{4}$ Center for Ergonomics, Industrial and Operations Engineering, University of Michigan, Ann Arbor, Michigan.

${ }^{5}$ Department of Emergency Medicine, University of Michigan Health System, Ann Arbor, Michigan.

${ }^{6}$ Correspondence should be directed to Robert Werner, Department of Physical Medicine and Rehabilitation, University of Michigan Health System, Ann Arbor, Michigan.
} 
industrial population and a range of $1.0-3.46$ per 1000 person-years in the general population (2,6-8).

Previous studies have identified potential risk factors for development of CTS that include ergonomic stressors (9), median mononeuropathy (10), body mass index (BMI) (11), gender $(7,12)$, diabetes $(13,14)$, and rheumatoid arthritis $(15,16)$, however, the majority of these studies have been cross-sectional studies or studies with very short follow-up. Previous studies by Werner et al. (17) and Nathan et al. (2) with longer follow-up times, 6 and 11 years respectively, found a relationship between baseline median mononeuropathy and subsequent development of CTS. Despite this association, more than $70 \%$ of those with nerve conduction slowing at baseline did not develop symptoms of CTS at follow-up thereby limiting the use of median mononeuropathy as a predictor of future development of CTS. The natural history of CTS is not well characterized due to the lack of longitudinal studies in this area. While associations between CTS and the risk factors listed above have been identified, longitudinal studies would better define these relationships.

The purpose of this study was to examine workers over an extended period of time to identify factors which influence the symptoms and physical findings associated with CTS and to create a predictive model of who is at risk for CTS. For this study we examined workers with no history of CTS at baseline, who subsequently developed symptoms and physical findings consistent with CTS (Incident Cases), and compared them to a group of workers who were never diagnosed with CTS (Controls).

We hypothesized that Incident Cases would be female, work in jobs with higher ergonomic stressors (hand activity level, peak force), have a higher prevalence of diabetes, and higher BMI. We also hypothesized that Incident Cases would be more likely to have a baseline median mononeuropathy. On the basis of the known association between nerve conduction slowing and aging we hypothesized that workers who went on to develop CTS would be older.

\section{METHODS}

This was a longitudinal study of workers from four industrial and three clerical work sites. Of the 985 subjects who participated in a baseline study, 501 (51\%) were screened an average of 5.4 years later. Excluding subjects that could not be contacted, there was a 74\% participation rate at follow-up. Eighteen percent $(n=179)$ declined to participate and $31 \%$ $(n=305)$ of the original 985 could not be contacted.

The baseline demographics of both participants and nonparticipants in the followup screening are presented in Table I. Nonparticipants did not differ with regards to BMI, hand dominance, repetition level, median mononeuropathy, or prevalence of upper extremity tendonitis compared to responders. Responders were significantly older than nonresponders ( 39.1 vs. $35.8, p<0.01$ ), were more likely to be female ( $71 \%$ vs. $62 \%, p=0.004$ ), had a significantly higher percentage reporting neck/shoulder symptoms $(49 \%$ vs. $43 \%, p=$ $0.04)$, and were more likely to have had a diagnosis of CTS at baseline (6\% vs. $3 \%$, $p=0.01)$.

Subjects were eligible to participate if they were in the same job, had changed jobs, or retired. All subjects underwent electrodiagnostic testing according to the techniques described by Kimura. All subjects underwent a directed physical examination of the upper extremities and completed a symptom questionnaire, including a hand diagram (18), at 
Table I. Comparison of Baseline Results of Responders vs. Nonparticipants to Recruitment at Time 2 (5-Year Follow-up). Mean (SD)

\begin{tabular}{lccc}
\hline & Responders $(n=501)$ & All nonresponders $(n=484)$ & $p$ \\
\hline Age [mean (SD)] & $39.1(9.9)$; range 19-69 & $35.8(10.5)$; range 19-65 & 0.00 \\
BMI [mean (SD)] & $28.3(6.6)$ & $27.6(6.5)$ & 0.09 \\
Baseline repetition level [mean (SD)] & $5.8(1.9)$ & $5.9(1.9)$ & 0.49 \\
Gender (\% female) & $71 \%$ & $62 \%$ & 0.00 \\
Hand dominance (right-hand dominant) & $10 \%$ & $11 \%$ & 0.51 \\
Median mononeuropathy ( $\geq 0.5$ ms) & $18 \%$ & $15 \%$ & 0.23 \\
Diabetes & $2.2 \%$ & $2.7 \%$ & 0.61 \\
Rheumatoid arthritis & $2.4 \%$ & $1.2 \%$ & 0.18 \\
Current neck/shoulder symptoms & $49 \%$ & $43 \%$ & 0.04 \\
Current elbow/forearm symptoms & $31 \%$ & $26 \%$ & 0.06 \\
Current wrist/hand/finger symptoms & $55 \%$ & $50 \%$ & 0.12 \\
Tendonitis in the upper extremity & $16 \%$ & $15 \%$ & 0.71 \\
CTS & $6 \%$ & $3 \%$ & 0.01 \\
\hline
\end{tabular}

both baseline and follow-up. All jobs were assessed and rated for ergonomic exposures at baseline. Each job was rated according to the American Congress of Governmental Industrial Hygienists' (ACGIH) Threshold Limit Values (TLV) for hand activity level based upon the hand repetition level and the normalized peak force (19). Psychosocial variables were assessed using a questionnaire based on the one developed by Karasek (20). The areas assessed included estimates of skill discretion, job insecurity, perceived stress and job satisfaction based on the decision latitude of the worker and the psychological demands placed upon the worker. Each worker was weighed and measured for height to calculate the body mass index (BMI, $\left.\mathrm{kg} / \mathrm{m}^{2}\right)$.

Subjects who had a previous history of CTS or were diagnosed with CTS at the first screening were not included in the analysis for this study. Incident cases were defined as workers who reported that a physician made the diagnosis of CTS since the time of the initial screening which resulted in carpal tunnel surgery or a worker who met our criteria for CTS at the follow-up. Our diagnosis of CTS was defined as numbness, tingling, burning, or pain in the distribution of the median nerve (based on a hand diagram score of "probable" or "definite") with ipsilateral median nerve conduction slowing (a median sensory peak latency difference of $\geq 0.5 \mathrm{~ms}$ compared to the ipsilateral ulnar sensory peak latency). Those workers who did not develop CTS were considered "controls."

\section{STATISTICAL ANALYSIS}

A univariate analysis using $t$ tests or chi-square analysis was performed on the two groups (Incident cases vs. Controls) comparing them for baseline demographics, medical history, ergonomic stresses, psychosocial factors, discomfort ratings, and electrophysiologic results. Multivariate logistical regression was performed using new onset of CTS as the dependent variable to create a predictive model based on the data from the initial screening.

\section{RESULTS}

Five hundred and one subjects, $51 \%$ of the 985 subjects from the original study, participated in the follow-up screenings. Of those 501 subjects, 69 had been diagnosed 
with CTS prior to the baseline screening by a medical provider or were diagnosed with CTS based on the screening results and therefore were eliminated from the analysis. Of the remaining 432 subjects, $29(7 \%)$ developed CTS in the interim between screenings. Twenty-two subjects were assigned a diagnosis of CTS at follow-up based on symptoms and nerve conduction results at the second screening plus an additional seven subjects who were diagnosed by their own physician and subsequently had carpal tunnel surgery for a total of 29 incident cases.

The average length of follow-up for subjects included in the analysis was 5.4 years. On the basis of the number of new cases over the 5.4 years, the cumulative incidence of CTS in this population was 12.4 cases per 1000 workers per year with an average incidence rate of $1.2 \%$ per year.

Compared to the 385 subjects who did not develop CTS, incident cases of CTS had significantly higher BMI (32.3 vs. 27.7, $p=0.0003$ ) and were more likely to have a previous diagnosis of tendonitis in the UE $(24 \%$ vs. $13 \%, p<0.07)$ (see Table II). Subjects who developed CTS were more likely to report symptoms in the wrist/hand/fingers at baseline

Table II. Comparison of Baseline Results in Those Who Developed CTS Between Screenings and Those Who Did Not

\begin{tabular}{|c|c|c|c|}
\hline & $\begin{array}{c}\text { Incident cases } \\
\text { of CTS } \\
(n=29)\end{array}$ & $\begin{array}{c}\text { Control subjects: } \\
\text { no CTS } \\
(n=403)\end{array}$ & $p$ \\
\hline \multicolumn{4}{|l|}{ Personal factors } \\
\hline BMI & 32.3 & 27.8 & 0.00 \\
\hline Age & 38.8 & 38.3 & 0.72 \\
\hline Gender (\%female) & $69 \%$ & $69 \%$ & 0.63 \\
\hline Wrist/hand/finger symptoms & $69 \%$ & $48 \%$ & 0.03 \\
\hline Wrist/hand/finger worst discomfort rating & 2.22 & 2.05 & 0.76 \\
\hline Hand diagram score $>0$ & $48 \%$ & $19 \%$ & 0.00 \\
\hline Any UE tendonitis & $32 \%$ & $11 \%$ & 0.00 \\
\hline Diabetes & $7 \%$ & $1 \%$ & 0.03 \\
\hline Rheumatoid arthritis & $4 \%$ & $2 \%$ & 0.45 \\
\hline Exercise & $65 \%$ & $61 \%$ & 0.70 \\
\hline Smoke & $38 \%$ & $41 \%$ & 0.74 \\
\hline Average length of follow-up (months) & 63.7 & 64.7 & 0.56 \\
\hline$\%$ in clerical jobs & $59 \%$ & $60 \%$ & 0.88 \\
\hline Retire & $7 \%$ & $9 \%$ & 0.66 \\
\hline Job change between screenings & $66 \%$ & $57 \%$ & 0.39 \\
\hline \multicolumn{4}{|l|}{ Psychosocial factors } \\
\hline Skill discretion & 23.4 & 25.2 & 0.23 \\
\hline Decision authority & 24.4 & 26.1 & 0.35 \\
\hline Job creativity & 6.5 & 6.8 & 0.39 \\
\hline Coworker support & 11.6 & 11.9 & 0.56 \\
\hline Supervisor support & 11.5 & 11.3 & 0.65 \\
\hline Job insecurity & 4.9 & 4.5 & 0.28 \\
\hline Job satisfaction & 0.33 & 0.31 & 0.54 \\
\hline Perceived stress & 26.7 & 22.9 & 0.02 \\
\hline \multicolumn{4}{|l|}{ Electrodiagnostic factors } \\
\hline Median mononeuropathy $\geq 0.5 \mathrm{~ms}$ & $48 \%$ & $12 \%$ & 0.00 \\
\hline Median ulnar peak latency difference (dominant side) & 0.67 & 0.12 & 0.00 \\
\hline \multicolumn{4}{|l|}{ Dominant hand ergonomic variables } \\
\hline Hand activity level (HAL) & 6.1 & 5.6 & 0.19 \\
\hline Peak force & 3.1 & 3.0 & 0.62 \\
\hline Threshold limit value $>2$ (Peak Force and HAL) & $32 \%$ & $23 \%$ & 0.26 \\
\hline
\end{tabular}


Table III. Logistic Regression Analysis: Modeling Predictors for Incident Cases of CTS

\begin{tabular}{lccc}
\hline \multicolumn{1}{c}{ Independent variables } & Odds ratio & $P>|t|$ & 95\% confidence interval \\
\hline BMI > 27 at baseline & 2.29 & 0.085 & 0.9 to 5.9 \\
Median ulnar peak latency difference (per 0.1 ms increase) & 1.29 & 0.000 & 1.2 to 1.4 \\
Numbness, tingling, burning, pain in the hand at baseline & 5.22 & .001 & 2.1 to 6.5 \\
TLV for peak force-Hand activity level =3 & 1.6 & .15 & .9 to 2.9 \\
\hline
\end{tabular}

Note. $n=414, p$ value for model $<0.001$.

(69\% vs. $48 \%, p=0.03$ ), however there was no difference in the level of discomfort ratings, among those who reported any discomfort, in the wrist/hand/fingers between the two comparison groups (2.22 vs. 2.05, $p=0.76)$. On the basis of a hand diagram score greater than $0,48 \%$ of those who developed CTS reported having numbness, tingling, burning, and/or pain in the distribution of the median nerve at baseline compared to $19 \%$ of those who never developed CTS $(p=0.001)$. Among the subjects who completed all of the psychosocial questions $(n=315)$, those who developed CTS had higher levels of perceived stress (26.7 vs. 22.9, $p=0.02$ ) but did not differ on any of the other psychosocial variables. No significant differences or trends were noted between groups with respect to age, gender, type of work (industrial vs. clerical), job change, or retirement between screenings. There was no difference in the average length of follow-up time between the two groups.

Individuals with baseline evidence of nerve dysfunction (median to ulnar latency difference $\geq 0.5 \mathrm{~ms}$ ) were more likely to be diagnosed with CTS at follow-up (45\% vs. $12 \%$, $p<0.01)$. The dominant hand median-ulnar peak latency difference at baseline was significantly higher for those subjects with incident cases of CTS $(0.67 \mathrm{~ms}$ vs. $0.10 \mathrm{~ms}, p<0.01)$.

While there was a trend for those with new onset of CTS to have jobs with greater hand activity level, the difference did not reach statistical significance. Those with incident CTS tended to work in jobs that exceeded the threshold limit value (TLV) for peak force/hand activity level to the "unacceptable" level (32\% vs. 23\%, $p=0.26)$ (see Table II). Other differences between the two groups comparing ergonomic stressors at baseline were not significant.

Multiple logistic regression using various demographic, electrophysiological, and ergonomic factors as independent variables yielded a useful model for predicting CTS (pseudo $R^{2}=0.25, p<0.0001$ ) (see Table III). Workers with baseline numbness, tingling, burning, and/or pain in the fingers were 5.2 times more likely to develop CTS. For each $0.1 \mathrm{~ms}$ increase in the median-ulnar peak latency difference, the relative risk for developing CTS increase by $29 \%$. Workers in jobs rated at a peak force-hand activity threshold limit value (TLV) of 3, or "unacceptable," were 1.6 times more likely to go on to acquire CTS compared to workers with a normal or borderline level but this did not reach statistical significance. There was a trend for a BMI $>27$ to double the risk of CTS but this did not reach statistical significance $(p=0.09)$. All other independent variables fell out of the regression model.

\section{DISCUSSION}

The estimated annual incident rate for CTS in this population was 12.4 per 1000 workers per year. This is higher than the range of annual incidence rates, 1.0 to 3.46 per 1000 workers, reported from retrospective studies of the general populations (6-8). While 
Franklin et al. (5) found an average incidence rate of 1.74 per 1000 workers per year, the incidence rates stratified by industrial classification ranged from 0.8 to 14.8 . Higher rates were associated with jobs considered to have more stressful workplace exposures (fish packing and processing, meat and poultry dealers, carpentry) compared to industries with typically less stressful workplace exposures (sales, restaurants, higher education institutions, and clerical). A large portion of our population was selected to include equal proportions of subjects from low, medium, and high repetition jobs. Our study population may have an overrepresentation of workers in high repetition jobs compared to the general population and probably even other industrial populations, thereby translating into a higher incident rate for CTS. Additionally, incident cases in the Franklin study were identified using workers' compensation (WC) records which probably underrepresent the true number of cases. Gerr et al. (21) found an annual incidence rate of 9 cases per 1000 person-years among computer users. This is higher than the incidence rate reported in other studies of clerical workers. Given that the subjects in the Gerr (21) study all worked in low ergonomic stressor jobs, it is not surprising that it is lower than our incidence rate among subjects from a range of high, medium, and low exposure jobs. Approximately 50\% of the original subjects were lost to follow-up at the second screening. Even if all of the subjects lost to follow-up remained healthy over the 5 years, our incident rate for CTS would still approach 7 per 1000 workers; this is higher than previously reported incident rates but closer to the rate found by Gerr et al. (21). It is possible that the heightened awareness of CTS in the general population in the last 5-10 years has led to earlier recognition and diagnosis compared to the years of the previous studies looking at incident cases of CTS.

Previous studies have identified risk factors for CTS but the natural history of occupational CTS, including predictors of CTS, has not been well described. The current study uses a longitudinal design to examine possible factors of CTS and symptoms consistent with CTS. As we hypothesized, baseline characteristics such as median mononeuropathy, and a job with an unacceptable TLV for hand activity (high peak force-high repetition) were associated with new onset of carpal tunnel syndrome within 5 years. We also hypothesized that a high BMI would be associated with new onset of CTS. This held true in the univariate analysis although BMI was not a significant contributor to the predictive logistic regression model (there was a trend suggesting that a BMI greater than 27 may influence new onset of CTS when controlling for other independent variables). A history of diabetes was a significant factor in the univariate analysis but dropped out of the logistic regression analysis. Both diabetes and rheumatoid arthritis (RA) have been identified as increasing the risk for developing CTS by 2.5-3.5 times (22,23). Given the low percentage of subjects in our study with diabetes and RA at baseline there may not have been enough subjects in our analysis to produce significant results.

Contrary to our expectations, age and gender were not significant in the univariate analysis nor did they contribute to the predictive model when controlling for other risk factors. Additionally, workers from the industrial setting were no more likely to develop CTS compared to clerical workers. Previous studies have reported an association between higher physical fitness or aerobic conditioning and lower risk for CTS (24), but we found no relationship between frequency of exercise and new onset of CTS. Previous populationbased studies have suggested that female gender is a risk factor for CTS, especially in those aged 50-70 (1,7,25). However our results are similar to those found in a study by Franklin et al. (2) which demonstrated that the female/male ratio for CTS in the general population decreases from $3: 1$ to $1.2: 1$ in the occupational setting. 
As expected, we saw a trend toward a greater percentage of subjects above the TLV for the integration of hand activity level and peak force, among the incident cases in the univariate analysis and in the predictive model. This suggests that the interaction of workrelated factors, such as peak force and hand activity level may be more influential on CTDs than the individual stressors.

Workers who went on to develop CTS were more likely to report wrist/hand/finger symptoms at baseline, and were more likely to have a previous diagnosis of tendonitis in the UE. Similar results were found in a recent nested case control study of risk factors for CTS in women where Ferry et al. (26) found a significant relationship between new onset of $\mathrm{CTS}$ and a previous history of another musculoskeletal complaint (adjusted OR $=1.98$; CI: $1.98,2.97)$. A possible explanation for this is that the worker uses compensatory strategies that translate into higher loads on the hand/wrist. Alternatively it may reflect a genetic predisposition to soft tissue/connective tissue failure. Another possible explanation lies in how medical diagnoses are assigned. In some cases the symptoms and presentation of tendonitis and CTS can overlap making it difficult to discriminate between the two diagnoses. Referred pain from CTS can present in the elbow or shoulder. The fact that $60 \%$ of those who went on to develop CTS reported symptoms (numbness, tingling burning, pain) at baseline in the distribution of the median nerve suggests that people may have symptoms prior to other physical evidence, such as nerve conduction slowing. Further research is needed to understand how intervention or treatment at this stage might prevent the development of CTS.

Similar to previous research $(10,17)$ this study showed that subjects who went on to develop CTS were more likely to have a median mononeuropathy compared to those who did not develop CTS. Logistic regression clarified this further, demonstrating that as the median to ulnar sensory peak latency differences increase, so does the risk for developing CTS. This finding held true even in a subset of subjects whose median ulnar peak latency difference was considered normal ( $<0.4 \mathrm{~ms})$ at baseline. Nathan et al. (10) found similar results in a longitudinal study of industrial workers where new development of CTS was linearly correlated with initial median nerve conduction slowing.

Limitations of the study also include methodological issues such as limited surveillance of both subjects and jobs, recruitment of subjects, and modeling of the exposure-response relationships. Our population was initially chosen for a cross-sectional study and our criteria for eligibility was employment in the same job for at least 6 months. Given the opportunity, with additional funding, to transform the cross-sectional study into a longitudinal study, we chose to follow the same population despite the limitations of not having a cohort of newly hired workers included in the baseline screening. Another weakness of the study was the lack of ergonomic analysis of jobs at follow-up. Although every effort was made to assign accurate ergonomic stressor ratings at follow-up, the methods were limited compared to the on-site analysis conducted at the initial screening. Forty percent of the follow-up study population remained in the same job analyzed at the baseline screening, however no documentation was collected of changes made to these jobs. Several researchers have suggested there is a greater risk for new onset of UEMSDs in the first 6 months on a new, higher risk job $(27,28)$. This can lead to a "healthy worker effect" in the remaining worker population, i.e., the workers who experience a problem on a difficult job leave and only the workers who are successful at these jobs remain in the study group. Given the criteria that all subjects in the initial cross-sectional study be employed in the same job for at least 6 months, our population did not include those who may be most vulnerable to new 
onset of UEMSDs. Ideally, a prospective study would include more frequent surveillance of study subjects. However, due to the limitations of funding and the timing of the funding, we were limited to two periods of data collection providing a snapshot view of subjects at both times. Another weakness of the study included the loss of $50 \%$ of the original sample and the finding of differences between the responders and the nonresponders for the follow-up screening. The workers lost to follow-up tended to be younger and were less symptomatic at baseline. We feel that a $74 \%$ participation rate of workers whom we were able to locate was exceptional, but the loss of half of the original sample leaves a potential for selection bias.

\section{CONCLUSIONS}

This study corroborates the findings from previous cross-sectional studies on the natural history of CTS and potential risk factors for development of CTS $(2,9,17)$. The results lend further evidence to the usefulness of the ACGIH TLV for physical stressors as guidelines to reduce exposure and prevent cumulative trauma disorders in the occupational setting. The results also suggest that factors which may be amenable to treatment, such as hand/finger symptoms and wrist/hand/finger tendonitis, are early predictors of CTS. Additional research is needed in the area to determine the influence of work restrictions and early treatment intervention on the prevention of CTS.

\section{ACKNOWLEDGMENTS}

Support for this research is provided by the National Institute on Disability and Rehabilitation Research of the United States Department of Education, Grant \#H133E980007, "Rehabilitation Engineering Research Center." The opinions contained in this publication are those of the grantee and do not necessarily reflect those of the United States Department of Education.

\section{REFERENCES}

1. Atroshi I, Gummesson C, Johnsson R, Ornstein E, Ranstam J, Rosen I. Prevalence of carpal tunnel syndrome in a general Population. JAMA 1999; 282(2): 153-158.

2. deKrom MC, Kester AD, Knipschild PG, Spaans F. Risk factors for carpal tunnel syndrome. Am J Epid 1990; 132(6): 1102-1110.

3. Franzblau A, Werner RA, Valle J, Johnston E. Workplace surveillance for carpal tunnel syndrome: A comparison of methods. J Occup Rehabil 1993; 3(1): 1-14.

4. Homan MM, Franzblau A, Werner RA, Albers JW, Armstrong TJ, Bromberg MB. Agreement between symptom surveys, physical examination procedures and electrodiagnostic findings for carpal tunnel syndrome. Scand J Work Environ Health 1999; 25(2): 115-124.

5. Franklin GM, Haug J, Heyer N, Checkoway H, Peck N. Occupational carpal tunnel syndrome in Washington state, 1984-1988. Am J Pub Health 1991; 81: 741-746.

6. Nordstrom DL, DeStefano F, Vierkant RA, Layde PM. Incidence of diagnosed carpal tunnel syndrome in a general population. Epidemiology 1998; 9: 342-345.

7. Stevens JC, Sun S, Beard CM, O'Fallon WM, Kurtland LT. Carpal tunnel syndrome in Rochester, Minnesota 1961-1980. Neurology 1988; 38: 134-138.

8. Mondelli M, Giannini F, Giacchi M. Carpal tunnel syndrome incidence in a general population. Neurology 2002; 58(2): 289-294. 
9. Bernard B, ed. Musculoskeletal disorders and workplace factors. Cincinnati, OH: National Institute for Occupational Health and Safety, US Department of Health and Human Services, 1997.

10. Nathan PA, Keniston RC, Myers LD, Meadows KD, Lockwood RS. Natural history of median nerve sensory conduction in industry: Relationship to symptoms and carpal tunnel syndrome in 558 hands over 11 years. Muscle Nerve 1998; 21(6): 711-721.

11. Werner RA, Albers JW, Franzblau A, Armstrong TJ. The relationship between body mass index and the diagnosis of carpal tunnel syndrome. Muscle Nerve 1994; 17: 632-636.

12. Swajian GR. Carpal tunnel syndrome: A five year study. J Am Osteop Assoc 1981; 81: 49-51.

13. Albers JW, Brown MB, Sima AA, Greene DA. Frequency of median mononeuropathy in patients with mild diabetic neuropathy in the early diabetes intervention trial (EDIT). Muscle Nerve 1996; 19: 140-146.

14. Leach RE, Odon JA. Systemic causes of the carpal tunnel syndrome. Postgrad Med 1968; 44: 127-131.

15. Barnes $\mathrm{C}$, Currey H. Carpal tunnel syndrome in rheumatoid arthritis: A clinical and electrodiagnostic survey. Ann Rheum Dis 1967; 26: 226-233.

16. Herbison G, Teng C, Martin J, Ditunno J. Carpal tunnel syndrome in rheumatoid arthritis. Am J Phys Med 1973; 52: 63-74.

17. Werner RA, Gell N, Franzblau A, Armstrong TJ. Prolonged median sensory latency as a predictor of future carpal tunnel syndrome. Muscle Nerve 2001; 24(11): 1462-1467.

18. Katz JN, Stirrat CR. A self-administered hand diagram for the diagnosis of carpal tunnel syndrome. J Hand Surg 1990; 15A: 360-363.

19. ACGIH Worldwide. 2002 Threshold limit values for chemical substances and physical agents in the work environment. 2002.

20. Karasek R, Brisson C, Kawakami N, Houtman I, Bongers P, Amick B. The Job Content Questionnaire (JCQ): An instrument for internationally comparative assessments of psychosocial job characteristics. J Occup Health Psych 1998; 3(4): 322-355.

21. Gerr F, Marcus M, Ensor C, Kleinbaum D, Cohen S, Edwards A, Gentry E, Ortiz D, Monteilh. A prospective study of computer users: I. Study design and Incidence of musculoskeletal symptoms and disorders. AJIM 2002; 41: 221-235.

22. Atecheson SG, Ward JR, Lowe W. Concurrent medical disease in work-related carpal tunnel syndrome. Arch Intern Med 1998; 158: 1506-1512.

23. Stevens JC, Beard CM, O'Fallon WM, Kurland LT. Conditions associated with carpal tunnel syndrome. Mayo Clin Proc 1992; 67: 541-548.

24. Nathan PA, Keniston RC. Carpal tunnel syndrome and its relation to general physical condition. Hand Clinics 1993; 9(2): 253-261.

25. Dieck GS, Kelsey JL. An epidemiologic study of the carpal tunnel syndrome in an adult female population. Prev Med 1985; 14(1): 63-69.

26. Ferry S, Hannaford P, Warskyj M, Lewis M, Croft P. Carpal tunnel syndrome: A nested case-control study of risk factors in women. Am J Epidemiol 2000; 151(6): 566-574.

27. Park RM, Krebs JM, Mirer FE. Occupational disease surveillance using disability insurance at an automotive stamping and assembly complex. J Occup Environ Med 1996; 38(11): 1111-1123.

28. Flowerdew RE, Bode OB. Tenosynovitis in untrained farm-workers. Brit Med J 1942; II(4264): 367. 